\title{
COMT val I58met and 5-HTTLPR Genetic Polymorphisms Moderate Executive Control in Cannabis Users
}

\author{
Antonio Verdejo-García*, , , ${ }^{2}$ Ana Beatriz Fagundo ${ }^{2}$, Aida Cuenca ${ }^{2,3}$, Joan Rodriguez ${ }^{2}$, Elisabet Cuyás ${ }^{2}$, \\ Klaus Langohr ${ }^{2,4}$, Susana de Sola Llopis ${ }^{2}$, Ester Civit ${ }^{2}$, Magí Farré ${ }^{2,5}$, Jordi Peña-Casanova ${ }^{6}$ and \\ Rafael de la Torre $2,7,8$ \\ 'School of Psychology and Psychiatry, Monash University, Melbourne, VIC, Australia; ${ }^{2}$ Human Pharmacology and Clinical Neurosciences Research \\ Group, Neuroscience Research Program, IMIM-Hospital del Mar Medical Research Institute, Parc de Salut Mar, Barcelona, Spain; ${ }^{3}$ Epidemiology \\ of Drugs of Abuse Research Group, Public Health and Epidemiology Research Program, IMIM-Hospital del Mar Medical Research Institute, \\ Barcelona, Spain; ${ }^{4}$ Department of Statistics and Operational Research, Universitat Politècnica de Catalunya, Barcelona, Spain; ${ }^{5}$ Universitat \\ Autònoma de Barcelona (UAB), Barcelona, Spain; ' Behavioral Neurology and Dementias Research Group, Neurosciences Research Program, \\ IMIM-Hospital del Mar Medical Research Institute, Barcelona, Spain; 7 Universitat Pompeu Fabra (CEXS-UPF), Barcelona, Spain; ${ }^{8}$ CIBER de \\ Fisiopatología de la Obesidad y Nutrición (CB06/03), CIBEROBN, Santiago de Compostela, Spain
}

\begin{abstract}
The adverse effects of cannabis use on executive functions are still controversial, fostering the need for novel biomarkers able to unveil individual differences in the cognitive impact of cannabis consumption. Two common genetic polymorphisms have been linked to the neuroadaptive impact of $\Delta$ 9-tetrahydrocannabinol (THC) exposure and to executive functions in animals: the catechol-Omethyltransferase (COMT) gene val I58met polymorphism and the SLC6A4 gene 5-HTTLPR polymorphism. We aimed to test if these polymorphisms moderate the harmful effects of cannabis use on executive function in young cannabis users. We recruited 144 participants: 86 cannabis users and 58 non-drug user controls. Both groups were genotyped and matched for genetic makeup, sex, age, education, and IQ. We used a computerized neuropsychological battery to assess different aspects of executive functions: sustained attention (CANTAB Rapid Visual Information Processing Test, RVIP), working memory (N-back), monitoring/shifting (CANTAB ID/ED set shifting), planning (CANTAB Stockings of Cambridge, SOC), and decision-making (lowa Gambling Task, IGT). We used general linear model-based analyses to test performance differences between cannabis users and controls as a function of genotypes. We found that: (i) daily cannabis use is not associated with executive function deficits; and (ii) COMT val I 58met and 5-HTTLPR polymorphisms moderate the link between cannabis use and executive performance. Cannabis users carrying the COMT val/val genotype exhibited lower accuracy of sustained attention, associated with a more strict response bias, than val/val non-users. Cannabis users carrying the COMT val allele also committed more monitoring/shifting errors than cannabis users carrying the met/met genotype. Finally, cannabis users carrying the 5-HTTLPR s/s genotype had worse IGT performance than s/s non-users. COMT and SLC6A4 genes moderate the impact of cannabis use on executive functions.

Neuropsychopharmacology (2013) 38, 1598-1606; doi:I0.1038/npp.2013.59; published online 27 March 2013
\end{abstract}

Keywords: cannabis; COMT; decision making; serotonin; shifting; sustained attention

\section{INTRODUCTION}

Cannabis is the most consumed illegal drug among youths between 14 and 25 years old, with prevalence rates and treatment demands steadily increasing over the past years (EMCDDA Annual Report, 2011; NSDUH National Survey, 2010). Cannabis-induced reinforcing effects are triggered by direct activation of cannabinoid CB1 receptors (located in frontal-cingulate-striatal regions), and indirect stimulation

* Correspondence: Dr A Verdejo-García, School of Psychology and Psychiatry, Monash University, Wellington Road, Melbourne, VIC 3800, Australia, Tel: +34 652 84I 09I, Fax: +34 958243749 , E-mail: antonio.verdejo@monash.edu

Received 30 October 2012; revised 20 February 2013; accepted 22 February 2013; accepted article preview online 28 February 2013 of striatal dopamine release (Camí and Farré, 2003). These brain networks and neurochemical systems are critical for the functioning of the executive control system, which is involved in the active maintenance, updating, and shifting of the higher order goals that guide adaptive efficient behavior. In accordance with different conceptual models, the executive system encompasses a range of dissociable functions, including sustained attention, working memory, response monitoring, and decision making (Robertson and Garavan, 2000; Robbins, 2007; Verdejo-Garcia and PerezGarcia, 2007), and these functions largely operate on monoamine systems (Barnes et al, 2011). The long-term effects of heavy cannabis use have been clearly associated with learning and memory deficits in tandem with hippocampal attrition (Grant et al, 2003; Yücel et al, 2008). However, the potentially adverse effects of cannabis 
use on executive functions are still controversial, with some studies showing significantly reduced performance of heavy users $v s$ controls in tests of sustained attention, response monitoring, or decision making (Fontes et al, 2011; Verdejo-García et al, 2007), but meta-analytic data yielding overall small or negligible effects (Grant et al, 2003). These controversial findings raise the possibility that moderator variables contribute to the presence or severity of executive deficits among cannabis users, with growing evidence showing that both earlier age at onset and heavier use are associated with poorer executive function (Bolla et al, 2002; Fontes et al, 2011) and greater frontostriatal deficits (Arnone et al, 2008; Zalesky et al, 2012). However, no studies to date have explored whether genetic variations in the monoamine systems that support frontostriatal networks and executive control relate to individual differences in cannabis-related executive deficits.

Animal models have demonstrated that the catechol-Omethyltransferase (COMT) gene modulates the harmful effects of cannabis use on the prefrontal and hippocampal brain structure (Behan et al, 2012) and working memory (ÓTuathaigh et al, 2010). An experimental challenge study has also shown that the COMT gene moderates the impact of THC on memory and sustained attention, such that individuals carrying the high-activity $\mathrm{val} / \mathrm{val}$ genotype are more likely to show cannabis-induced cognitive deficits (Henquet et al, 2009). In healthy individuals, the neurobiological correlates of working memory encoding suggest an abnormally increased activation of the prefrontal-parietalstriatal network in val vs met carriers. As this network is also abnormally hyperactivated among cannabis users when performing working memory tests (Jager et al, 2006, 2010), it is plausible to hypothesize that carrying the val variant and using cannabis may have cumulative negative effects on working memory and sustained attention. Considerably less is known about cannabis $\times C O M T$ gene interactive effects on other executive functions, but there is consistent evidence from animal and human studies showing that the met/met genotype of this gene is associated with poorer response monitoring/shifting skills (Nolan et al, 2004; Tunbridge et al, 2004).

On top of cannabinoid and dopamine effects, there is also animal evidence showing that high doses of cannabinoid agonists induce reduction of platelet serotonin concentrations, accompanied by alterations of the functioning of the serotonergic system in cannabis chronic smokers (Velenovská and Fisar, 2007). The 5-HTTLPR polymorphism is one of the main regulators of serotonin transporter function (Greenberg et al, 1999), and the $s / s$ genotype (associated with lower serotonin levels) has been consistently associated with poor decision-making skills in animals and humans (van den Bos et al, 2009; He et al, 2010; Homberg et al, 2008; Sonuga-Barke et al, 2011). The 5-HTTLPR polymorphism may be specifically associated with decision-making skills due to its prominent role in emotional processing, and its link with the ventromedial prefrontal cortex-amygdala network (Bevilacqua and Goldman, 2011), which is also impacted by heavy cannabis use (Vaidya et al, 2011). There is evidence that higher THC concentrations predict poorer decision-making quality in regular users (Hermann et al, 2009), such that in this case, along the same lines, it is plausible that carrying the $s / s$ variant and using cannabis may have cumulative negative effects on decision-making skills.

The aims of this study were: (i) to assess the executive functions of young daily cannabis users compared with nondrug-using controls, also exploring potential effects of age at onset and use severity; and (ii) to investigate possible interaction effects of cannabis use and COMT val158met and 5-HTTLPR polymorphisms in explaining executive function performance in cannabis users. We hypothesized that: (i) cannabis users would have worse executive function performance than controls; (ii) cannabis users carrying the val/val genotype of the COMT val158met polymorphism would have worse working memory and sustained attention, whereas cannabis users carrying the met/met genotype would have worse monitoring/switching; and (iii) cannabis users carrying the $s / s$ genotype of the 5-HTTLPR polymorphism would have poorer decision-making performance.

\section{MATERIALS AND METHODS}

\section{Participants}

In all, 144 European-Caucasian participants: 86 cannabis users and 58 non-drug users (controls) participated in the study. The demographic and drug-use characteristics from both groups are summarized in Table 1.

Inclusion criteria for cannabis users were: daily use of cannabis ( $>7$ joints per week) for a minimum duration of 3 years, meeting Diagnostic and Statistical Manual IV (DSM-IV) criteria for cannabis abuse or dependence, age between 18 and 30 years old, and having at least basic-level education ( $>8$ years of education). The two latter inclusion criteria (and exclusion of the first two) were applied to controls. Volunteers were recruited through several sources: 'word of mouth', advertisement in the local university, and advertisement in the website of a local Non-Governmental Organization (Energy Control) specialized in providing harm reduction guidelines among drug users. All assessments were conducted in research-dedicated premises of the Clinical Research Unit (CRU) of IMIM-Hospital del Mar Research Institute.

Potential participants were medically screened at the CRU to rule out the presence of physical or neurological illness and of drug-related acute effects or withdrawal symptoms, as determined by standard physical examinations and biochemical determinations (supervised by the medical director). They were also carefully assessed to diagnose comorbid psychiatric disorders using a well-validated interview, the Psychiatric Research Interview for Substance and Mental Disorders (PRISM; Torrens et al, 2004), which provided DSM-IV diagnoses. In addition, the PRISM provided diagnoses for the whole spectrum of DSM-IV substance-use disorders, such that we were able to rule out the potential participants meeting the criteria for abuse or dependence on drugs different than cannabis. Therefore, all potential participants having medical illnesses or comorbid psychiatric/substance-use disorders (current or during the prior year) were excluded from the study. For non-drug user controls, additional exclusion criteria-based on the toxicological history-were the use of any illegal drug during the past year or in more than 10 occasions during lifetime. Toxicological history in the past 6 months was 
Table I Descriptive Data on Demographic Variables and Drug-Use Characteristics

\begin{tabular}{|c|c|c|c|}
\hline & $\begin{array}{c}\text { Cannabis } \\
(n=86) \\
\text { Mean (SD) }\end{array}$ & $\begin{array}{c}\text { Control } \\
(n=58) \\
\text { Mean (SD) }\end{array}$ & SMD $^{\mathbf{a}}$ \\
\hline Age & $21.5(2.9)$ & $23.2(4.8)$ & 0.44 \\
\hline Vocabulary WAIS-III & $11.3(2.0)$ & $12.6(1.9)$ & 0.67 \\
\hline Gender & & & 0.08 \\
\hline Male & $61(70.9)$ & $39(67.2)$ & \\
\hline Female & $25(29.1)$ & $19(32.8)$ & \\
\hline University degree, n (\%) & & & 0.55 \\
\hline Yes & $55(64.0)$ & $50(87.7)$ & \\
\hline No & $31(36.0)$ & $7(12.3)$ & \\
\hline Employment status, n (\%) & & & 0.15 \\
\hline Employed & $24(28.2)$ & $22(38.6)$ & \\
\hline Unemployed & $23(27.1)$ & II (19.3) & \\
\hline Student & $38(44.7)$ & $24(42.1)$ & \\
\hline Smoker, n (\%) & & & 1.15 \\
\hline Current smoker & $57(66.3)$ & $9(\mid 6.1)$ & \\
\hline Non-smoker/ex-smoker & $29(33.3)$ & $47(83.9)$ & \\
\hline Age at first tobacco use, years & $18.9(3.0)$ & $19.0(4.2)$ & 0.03 \\
\hline Cigarettes per day & $9.4(6.2)$ & $6.6(5.6)$ & 0.46 \\
\hline Age at first alcohol use, years & $14.8(1.4)$ & $15.6(1.4)$ & 0.59 \\
\hline Years of alcohol consumption & $6.7(3.0)$ & $7.6(4.4)$ & 0.21 \\
\hline Age at first cannabis use, years & $15.3(1.5)$ & & \\
\hline Years of cannabis use & $6.2(2.8)$ & & \\
\hline $\begin{array}{l}\text { Lifetime cannabis use (number of } \\
\text { joints) }\end{array}$ & $5645.1(3908.6)$ & & \\
\hline $\begin{array}{l}\text { Cannabis consumption during the } \\
\text { past year }\end{array}$ & $1002.2(678.6)$ & & \\
\hline
\end{tabular}

${ }^{a}$ Absolute value of standardized mean difference.

confirmed by hair testing (de la Torre et al, 2010; Pichini et al, 2006). These hair samples were also used to measure cannabidiol (CBD) and THC ratio concentrations pertaining to the last month of cannabis use by GC/MS (Falcón et al, 2011).

\section{Test Procedure}

This study was approved by and conducted in accordance with the local ethics committee (CEIC-Parc de Salut Mar). Upon arrival to the research centre (IMIM-Hospital del Mar Medical Research Institute), participants were informed of the ensuing protocol and provided informed consent before participating in the study. All participants were subjected to an initial medical exploration that included a detailed medical history, biochemical analyses, physical examination, urine and hair drug testing, and a brief neurological examination. Eligible subjects underwent a psychiatric interview to exclude the presence of major psychiatric disorders, history of abuse, or drug dependence (except for nicotine dependence) administered by a clinical psychologist. Afterwards, all participants were requested to observe a 72-h abstinence period (possible withdrawal effects were medically controlled), and urine and hair drug screens were carried out by immunoassay (CEDIA; Thermo Fisher Scientific) to confirm abstinence before neuropsychological testing. Drug classes screened for included: cannabis, MDMA, cocaine, opioids, amphetamine, and methamphetamine. All participants meeting the inclusion criteria underwent the neuropsychological assessment session of $120 \mathrm{~min}$. All subjects were economically compensated for their participation (80 euros per participant).

\section{Genotyping}

Genomic DNA was extracted from the peripheral blood leukocytes of all the participants using Flexi Gene DNA kit (Qiagen Iberia SL, Sevilla, Spain) according to the manufacturer's instructions.

The COMT val108/158met (rs4680) single-nucleotide polymorphism (SNP) allelic variants were determined using the 59 exonuclease TaqMan assay with ABI 7900HT Sequence Detection System (Real Time PCR) supplied by Applied Biosystems (Foster City, CA). Primers and fluorescent probes were obtained from Applied Biosystems (TaqMan SNP Genotyping assays: assay ID C_2255335_10). Reaction conditions were those described in the ABI PRISM $7900 \mathrm{HT}$ user's guide. Endpoint fluorescent signals were detected on the ABI 7900, and the data were analyzed using the SDS software, version 2.3 (Applied Biosystems).

$5 H T T L P R$ genotyping was performed using PCR as described by Cuyas et al. (2011). The reaction mixture contained: $1 \times$ PCR Amplification buffer and $1 \times$ PCR Enhancer solution (Invitrogen, Carlsbad, CA), $1.5 \mathrm{mM}$ $\mathrm{MgSO}_{4}, 300 \mathrm{mM}$ dNTPs, $0.5 \mathrm{pmol}$ of each primer, $0.5 \mathrm{U}$ of Taq DNA polymerase (Invitrogen) and $50 \mathrm{ng}$ of genomic DNA as template. The products of allelic-specific amplifications (allele L, $528 \mathrm{bp}$; allele S, $484 \mathrm{bp}$ for 5-HTTLPR) were detected on an automatic ABI 3730XL capillary sequencer and analyzed by the GeneMapper Software v.3.5 (Applied Biosystems).

Table 2 shows the genotype distribution for COMT val158met and 5-HTTLPR polymorphisms as a function of group (cannabis users and controls).

\section{Neuropsychological Assessment}

We implemented a computerized neuropsychological assessment aimed to target specifically five different aspects of executive control using well-validated probes from the CANTAB (Robbins et al, 1994, 1998, see www.camcog.com) and other sources. The domains and tests used were: sustained attention (CANTAB Rapid Visual Information Processing Test, RVIP), working memory (N-back; Watter et al, 2001), response monitoring/set shifting (CANTAB Intradimensional/Extradimensional set shifting, ID/ED), planning/reflection impulsivity (CANTAB Stockings of Cambridge, SOC), and decision making (Iowa Gambling Task, IGT; Bechara et al, 2000). The main performance indices obtained from the different probes were the RVIP probability of hit (number of hits/(number of hits + number of misses)) indexing accuracy of vigilance/sustained attention; the 
Table 2 Genotype Distributions of Participants in the Cannabis User and Control Groups

\begin{tabular}{lccc}
\hline & $\begin{array}{c}\text { Cannabis }(\boldsymbol{n}=\mathbf{8 6}) \\
\boldsymbol{n}(\mathbf{M} / \mathbf{F})\end{array}$ & $\begin{array}{c}\text { Control }(\mathbf{n}=\mathbf{5 8}) \\
\boldsymbol{n}(\mathbf{M} / \mathbf{F})\end{array}$ & P-value \\
\hline 5HTTLPR & & & 0.88 \\
L/L & $22(15 / 7)$ & $16(1 / / 5)$ & \\
L/S & $47(36 / 1)$ & $31(21 / / 0)$ & \\
S/S & $17(10 / 7)$ & $11(7 / 4)$ & \\
COMT val I58met & & & 0.09 \\
Val/val & $16(9 / 7)$ & $19(13 / 6)$ & \\
Val/met & $58(46 / 12)$ & $29(20 / 9)$ & \\
Met/met & $12(6 / 6)$ & $10(6 / 4)$ & \\
\hline
\end{tabular}

Abbreviation: $M / F$, male/female distribution.

Hardy-Weinberg equilibrium:

- 5 HTTLPR: cannabis, $P=0.5 \mathrm{I} 5$; controls, $P=0.793$.

- COMT val I 58met: cannabis, $P=0.0022$; controls, $P=1$.

$\mathrm{N}$-back hit rate (proportion of hits minus proportion of errors) indexing working memory accuracy; the ID/ED total number of errors (adjusted by number of stages completed) indexing performance monitoring errors; the SOC initiation time and number of movements to correct, indexing reflection-impulsivity and planning skills, respectively; and the IGT net score ((sum of advantageous deck choices $(C+D)$ minus sum of disadvantageous deck choices $(A+B)$ ) indexing quality of decision making. As both the $\mathrm{N}$-back and the IGT tasks involve multiple series of trial blocks, the performance measures were calculated for N-back blocks 1, 2, and 3 (100 trials each), which index increasing working memory loads, and for IGT blocks 1-5 (20 trials each), which index progressive decision-making learning phases: pre-hunch (blocks 1 and 2), hunch (block 3), and explicit decision phases (blocks 4 and 5) (Bechara et al, 2005).

The RVIP decision-making pattern was further explored using the $\mathrm{A}^{\prime}$ (discrimination) and $\mathrm{B}^{\prime}$ (response bias) parameters derived from signal detection theory; the first indexing the ability to detect target sequences, and the second reflecting the tendency to respond, regardless of whether the target is present. The ID/ED error pattern was also further analyzed by calculating both intradimensional (indexing learning) $v s$ extradimensional (indexing shifting) errors.

We also calculated a $Z$-scored total cognitive index by calculating and adding the standardized scores from the five main performance indices: RVIP probability of hit, N-back hit rate (in the harder 3-back condition), ID/ED total number of errors, SOC number of movements to correct, and IGT net score.

In addition to these tests, we used the vocabulary subtest from the Wechsler Adult Intelligence Scale as a proxy of verbal IQ.

\section{Statistical Analyses}

Sample characteristics are described by means of either mean and standard deviation (numerical variables) or absolute and relative frequencies (categorical variables). To quantify the differences between both study groups (cannabis users and controls), the standard mean difference was computed. The $\chi^{2}$-test was applied to study the association between cannabis consumption and the COMT val158met and 5-HTTLPR polymorphisms. In addition, it was used to check whether the Hardy-Weinberg equilibrium holds among both study populations. We used ANCOVA models (controlling for age, gender, estimated verbal IQ, and smoking status) to compare cannabis users and non-user controls, and to compare cannabis users with early $v s$ later onset of cannabis use (before $v s$ after 15 years old; Fontes et al, 2011). Pearson's correlation analyses were used to explore the association between estimates of cannabis use and THC/CBD concentrations and tests performance. Separate ANCOVA models were fitted to study the relation between cognitive performance and both cannabis consumption and the polymorphisms of interest while controlling for the covariates with significant impact on dependent measures. These models included study group and the respective genotype as predictive variables of interest as well as gender, age, estimated verbal IQ, and smoking status as potential covariates. Initially, all models did also include the two-way interaction between genotype and drug consumption. Whenever the interaction could be discarded, both factors were studied separately using the ANCOVA model excluding interaction. If, by contrast, the interaction was significant, the effect of drug consumption was studied separately for each genotype expression. In that case, the Tukey test for multiple comparisons was applied for the comparison of genotypes in the framework of the ANCOVA models including interaction. Statistical significance was set at 0.05 . The statistical software package $R$ (The $\mathrm{R}$ Foundation for Statistical Computing), version 2.13.1, was used for all analyses.

\section{RESULTS}

\section{Group Comparisons}

We found no significant differences between cannabis users and controls in any of the tasks' main performance indices or in the $Z$-scored cognitive score (see Table 3 ). We only found a significant difference between the groups on IGT block 3, which is considered to index the 'hunch' or learning phase of the task. The segregation of the cannabis group as a function of age at onset yielded the same pattern of results, and both subgroups of cannabis users (early and late onset) showed nonsignificant performance differences with controls.

\section{Correlations}

We did not find any significant correlation between selfreport lifetime estimates of cannabis use and performance in executive tests. However, we found a significant positive correlation between $\mathrm{CBD} / \mathrm{THC}$ ratio concentrations and SOC mean number of movements to correct, $r=0.52$; $95 \%$ CI: $0.31,0.68(P<0.001)$.

\section{Genotype $\times$ Group interactions}

Cannabis use $\times$ COMT val158met. Results showed a significant drug $\times$ gene interaction on RVIP probability of 
Table 3 Neuropsychological Performance as a Function of DrugUse Status: Cannabis Users vs Controls

\begin{tabular}{|c|c|c|c|c|}
\hline & $\begin{array}{c}\text { Cannabis } \\
(n=86)\end{array}$ & $\begin{array}{l}\text { Control } \\
(n=58)\end{array}$ & $\begin{array}{c}F \\
(I \mid 38)^{a}\end{array}$ & $P$-value ${ }^{a}$ \\
\hline \multicolumn{5}{|l|}{ Rapid visual processing } \\
\hline Probability of hit & $67.6(17.3)$ & $72.6(13.6)$ & 0.005 & 0.94 \\
\hline Alpha & $0.92(0.05)$ & $0.93(0.04)$ & 0.029 & 0.87 \\
\hline Beta & $0.93(0.09)$ & $0.91(0.09)$ & 0.965 & 0.33 \\
\hline \multicolumn{5}{|l|}{ N-back } \\
\hline $\begin{array}{l}\text { Probability of hit, } \\
N=1\end{array}$ & $90.7(10.7)$ & $93.3(8.8)$ & 0.189 & 0.66 \\
\hline $\begin{array}{l}\text { Probability of hit, } \\
N=2\end{array}$ & $81.5(15.0)$ & $83.9(16.2)$ & 0.019 & 0.89 \\
\hline $\begin{array}{l}\text { Probability of hit, } \\
N=3\end{array}$ & $60.9(23.4)$ & $65.0(23.9)$ & 0.001 & 0.97 \\
\hline \multicolumn{5}{|c|}{ Intralextradimensional set shifting } \\
\hline Total errors adjusted & $25.8(21.8)$ & $22.4(19.7)$ & 0.037 & 0.85 \\
\hline $\begin{array}{l}\text { Intradimensional } \\
\text { errors }\end{array}$ & $7.92(5.43)$ & $8.19(6.37)$ & 0.347 & 0.56 \\
\hline $\begin{array}{l}\text { Extradimensional } \\
\text { errors }\end{array}$ & $10.86(\mid 1.07)$ & $9.08(9.79)$ & 0.146 & 0.70 \\
\hline \multicolumn{5}{|l|}{ Stockings of Cambridge } \\
\hline $\begin{array}{l}\text { Mean choices to } \\
\text { correct }\end{array}$ & $1.4(0.3)$ & $1.3(0.2)$ & 0.601 & 0.44 \\
\hline $\begin{array}{l}\text { Mean latency to first } \\
\text { choice }\end{array}$ & $14249(4936)$ & $16247(5808)$ & 2.427 & 0.12 \\
\hline \multicolumn{5}{|l|}{ lowa Gambling Test } \\
\hline Block I & $-2.8(4.4)$ & $-2.7(4.3)$ & 0.182 & 0.67 \\
\hline Block 2 & $0.3(5.4)$ & $0.5(4.6)$ & 0.272 & 0.60 \\
\hline Block 3 & I.I (6.2) & $3.6(7.7)$ & 6.339 & 0.01 \\
\hline Block 4 & $2.4(7.2)$ & $4.0(8.8)$ & 2.554 & 0.11 \\
\hline Block 5 & $3.2(7.8)$ & $5.0(9.17)$ & 1.728 & 0.19 \\
\hline Net score & $4.56(21.5)$ & I0.33 (24.1) & 3.549 & 0.06 \\
\hline$Z$ composite score & $-0.48(2.97)$ & $0.7 \mid(2.94)$ & 1.004 & 0.32 \\
\hline
\end{tabular}

Results are presented as mean (SD).

${ }^{a}$ From ANCOVA models adjusting for sex, age, smoking status, and IQ.

hits, $\mathrm{F}(1,138)=5.17, P<0.05$, and ID/ED total number of errors, $\mathrm{F}(1,138)=6.06, P<0.05$. Planned paired contrasts showed that, in the case of RVIP, the interaction was driven by lower probability of hits in cannabis users carrying the val/val genotype (see Table 4 and Figure 1); val/val cannabis users performed significantly worse than $\mathrm{val} / \mathrm{val}$ controls $(P=0.002)$. Additional analyses of the RVIP signal detection parameters showed that val/val cannabis users exhibited significantly higher response bias $\left(\mathrm{B}^{\prime}\right)$ scores than $v a l / v a l$ non-users $(P=0.02)$. Conversely, their discrimination scores $\left(\mathrm{A}^{\prime}\right)$ were lower, although this difference was not significant. No differences were found for met carriers. In the case of ID/ED, the interaction was driven by the significant difference between cannabis users carrying the
Table 4 Neuropsychological Performance as a Function of Gene $\times$ Study Group Interaction

\begin{tabular}{|c|c|c|c|c|}
\hline & $\begin{array}{c}\text { Cannabis } \\
(n=86)\end{array}$ & $\begin{array}{l}\text { Control } \\
(n=58)\end{array}$ & $\begin{array}{c}F \\
(1,136)^{a}\end{array}$ & $P$-value \\
\hline \multicolumn{5}{|l|}{ RVP: probability of hit } \\
\hline COMT vall 58met & & & 5.17 & 0.02 \\
\hline Vallval & $61.6(14.9)$ & $76.6(11.4)$ & & \\
\hline Val/met and met/met & $69.0(17.6)$ & $70.7(14.2)$ & & \\
\hline \multicolumn{5}{|l|}{ RVP: alpha } \\
\hline COMT vall 58met & & & 2.93 & 0.09 \\
\hline Vallval & $0.90(0.04)$ & $0.94(0.03)$ & & \\
\hline Val/met and met/met & $0.92(0.05)$ & $0.92(0.04)$ & & \\
\hline \multicolumn{5}{|l|}{ RVP: beta } \\
\hline COMT val I 58met & & & 4.16 & 0.04 \\
\hline Vallval & $0.95(0.05)$ & $0.88(0.12)$ & & \\
\hline Val/met and met/met & $0.92(0.10)$ & $0.93(0.08)$ & & \\
\hline \multicolumn{5}{|l|}{ IED total errors adjusted } \\
\hline COMT val I58met & & & 6.06 & 0.01 \\
\hline Val/val and val/met & $28.0(22.6)$ & $21.0(19.1)$ & & \\
\hline Met/met & $12.3(6.5)$ & $29.1(22.0)$ & & \\
\hline \multicolumn{5}{|l|}{ IED extradimensional errors } \\
\hline COMT vall 58met & & & 6.51 & 0.01 \\
\hline Vallval and val/met & $11.6(11.3)$ & $7.7(9.01)$ & & \\
\hline Met/met & $6.6(6.5)$ & $15.6(1 \mid .3)$ & & \\
\hline \multicolumn{5}{|l|}{ lowa Gambling Test } \\
\hline 5-HTTLPR & & & 3.78 & 0.05 \\
\hline$L / /$ and I/s & $6.1(21.4)$ & $8.0(23.5)$ & & \\
\hline $\mathrm{S} / \mathrm{s}$ & $-1.5(21.3)$ & $20.2(25.3)$ & & \\
\hline
\end{tabular}

Results are presented as mean (SD).

a $P$-value of interaction term in ANCOVA model adjusting for sex, age, smoking status, and IQ, with the exception of lowa Gambling Task, in which age and smoking status were not associated with performance.

val allele and cannabis users carrying the met/met genotype $(P=0.02)$, the former showing a higher number of errors (Table 4). The detailed analysis of intra- $v s$ extradimensional errors showed that differences were driven by extradimensional or shifting errors (Table 4).

The N-back task was analyzed using a 'group $\times$ genotype $\times$ working memory load' mixed model. Although the interaction effect was not significant, $\chi^{2}(1,138)=2.08$, $P=0.14$, visual inspection of the data shows that performance mildly varies between groups as a function of genotype and load: whereas controls carrying different genotypes have a uniform drop of performance as a function of load, met/met cannabis users have a smoother drop of performance as a function of load with respect to val carriers (see Supplementary Figure 1).

We found no drug $\times$ gene interaction effects on the SOC, the IGT, or the total cognitive index. 
Cannabis use $\times 5$-HTTLPR. Results showed a significant drug $\times$ gene interaction on IGT, $\mathrm{F}(1,138)=3.78, P=0.05$. Planned paired contrasts showed that the interaction was driven by significant differences between cannabis users and controls carrying the $s / s$ genotype (see Table 4 and Figure 2); $s / s$ cannabis users performed significantly worse than $s / s$ controls $(P=0.02)$ (see Table 4$)$. To further explore decision-making learning across the task, we analyzed the 'group $\times$ block' interaction using a mixed model. The analysis showed evidence of an interaction effect, $\chi^{2}(1,138)=3.59, P=0.05$, and paired comparisons showed this effect was driven by the significant differences between $s / s$ cannabis users and $s / s$ controls $(P=0.01)$. Visual inspection of the group by block learning pattern show that, whereas $s / s$ controls have a steep learning gain across blocks, $s / s$ cannabis users take longer to learn the task (performance still $<0$ by block 4 ) (see Figure 2). No differences were found for $l$ carriers.

We found no drug $\times$ gene interaction effects on the N-back, RVIP, ID/ED, SOC, or total cognitive index.

\section{DISCUSSION}

This study yielded two major findings: (i) daily cannabis consumption does not significantly impair executive functions among young users, not even in users with age of onset before 15 years old; but (ii) COMT val158met and 5-HTTLPR genetic polymorphisms moderate the associa-

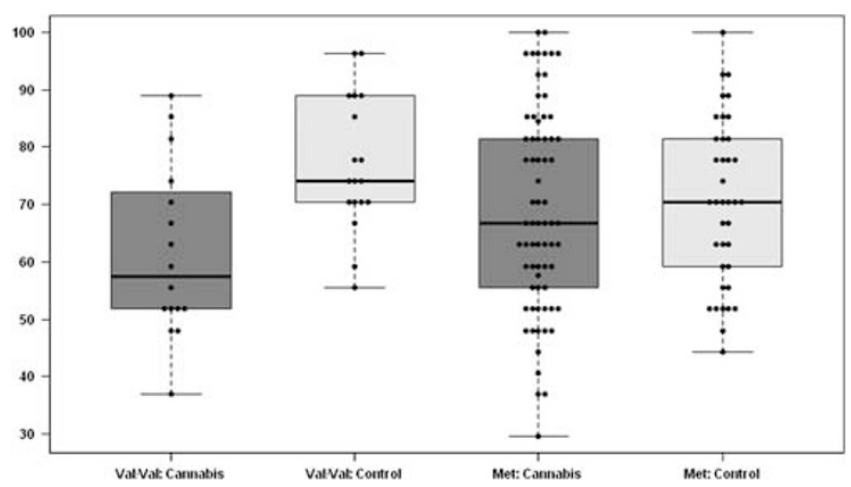

Figure I Rapid visual information processing's proportion of hits as a function of catechol-O-methyltransferase (COMT) gene and group. tion between cannabis use and executive functions. Specifically, cannabis users carrying the $\mathrm{val} / \mathrm{val}$ genotype of the COMT val158met gene exhibited lower accuracy of sustained attention associated with a more strict response bias than non-users carrying the same genotype. The cannabis users carrying the val allele also committed more monitoring/shifting errors than cannabis users carrying the met/met genotype. Finally, cannabis users carrying the 5 -HTTLPR $s / s$ genotype had worse decision-making skills than their non-user peers with the same genotype. Therefore, results indicate that certain genotypes of the COMT and the SLC6A4 genes strengthen the impact of cannabis use of different executive functions.

With respect to the first finding, the absence of general adverse effects of cannabis use on executive functions is consistent with the outcomes from the most exhaustive meta-analyses conducted so far about the link between cannabis and cognition (Grant et al, 2003). They concluded that the main adverse effect of cannabis use on cognitive function was restricted to the domains of learning and memory, whereas effects were not significant for the attention/ executive control domain, which we addressed here. Previous studies have argued that the adverse effects on cannabis use on executive function may be dose-related (Bolla et al, 2002). Partially in agreement with this notion, we showed that the CBD/THC hair concentration correlated with a higher number of movements to achieve the correct response in the SOC, pointing to a mild dose-dependent effect of cannabis use on planning skills. Planning deficits have been previously reported as long-term effects of cannabis use in a remarkably well-controlled twin study, in which non-cannabis user twins served a control group for their cannabis user siblings (Lyons et al, 2004). Nonetheless, the overall results extend the notion that regular cannabis use is not substantially harmful for executive control among daily users. A complementary notion, which we further tested, is whether individual differences in genetic makeup may place only certain cannabis users at higher risk of developing executive impairment.

According to our second finding, there are substantial moderation effects of the COMT val158met and the 5-HTTLPR genetic polymorphisms on the potentially harmful effects of cannabis use on particular components of executive control. Importantly, these moderation effects are biologically plausible and in agreement with previous knowledge about the influence of these polymorphisms on
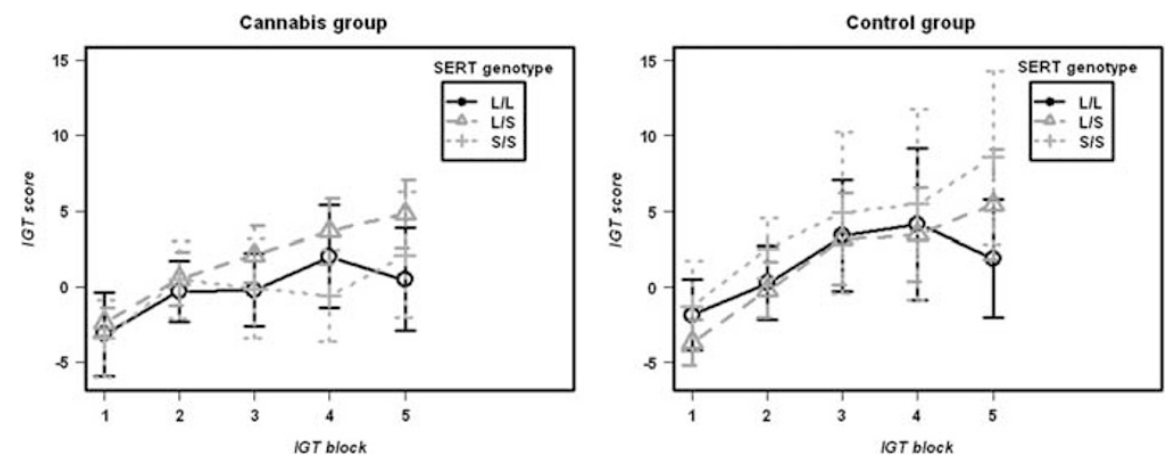

Figure 2 Block by block lowa Gambling Task's net scores as a function of serotonin transporter (SERT) gene and group. 
executive mechanisms. With regard to the COMT val158met polymorphism, we found that cannabis users carrying the $\mathrm{val} / \mathrm{val}$ genotype exhibited lower accuracy in detecting target sequences during sustained attention, and more strict response bias (less proneness to respond). This genotype has been associated with altered functioning of dopamine richly innervated prefrontal-parietal networks (Tan et al, 2007), and more lapses during sustained attention in healthy volunteers (Hamidovic et al, 2010). On the side of cannabis use, animal models have demonstrated that cannabinoid agonists significantly increase omission errors and response latencies (but not commission errors) in the 5-choice serial reaction time task (5CSRTT) (Pattij et al, 2008), which is an animal analog of the continuous performance test used here (Chamberlain et al, 2011). Further evidence has shown that administration of dopamine D1 partial agonists and D2/D3 antagonists produce similar effects on the 5CSRTT, impacting sustained attention by increasing omission (or vigilance) errors (Barnes et al, 2012; Besson et al, 2010). These findings are in agreement with our results, which show that the COMT val/val genotype (associated with lower dopamine availability) and cannabis use (potentially impacting dopamine function) interactively impact vigilance accuracy in the context of a stricter response bias, which mainly promotes omission errors. Whereas the val/val genotype has been associated with abnormally lower tonic dopamine functioning, the other monozygotic variant (met/met) has been linked to reduced phasic dopamine turnover (Bilder et al, 2004), and set-shifting decrements in both animal and human studies (Nolan et al, 2004; Tunbridge et al, 2004). Our results replicated these patterns in the case of healthy individuals (met/met carriers committed more shifting errors than $\mathrm{val} / \mathrm{val}$ carriers). However, the pattern of performance was reversed in cannabis users, in which the val carriers showed significantly higher errors than met/met cannabis carriers. This reversed pattern is consistent with structural imaging data of the COMT gene $\times$ cannabis use interaction in regions relevant for stimulus-outcome learning and shifting, as we have recently shown that the val allele is associated with smaller ventral caudate volumes in cannabis users, whereas the opposite is true for controls (Batalla et al, 2012).

A second cannabis $\times$ gene interaction effect was found for the link between cannabis use and decision making, moderated by the 5-HTTLPR polymorphism: cannabis users carrying the $s / s$ genotype took longer to learn and performed significantly more poorly the IGT than $s / s$ control carriers. This effect is consistent with the association between the $s / s$ genotype and poorer IGT performance, especially during initial phases of the task (van den Bos et al, 2009; He et al, 2010; Homberg et al, 2008), in which feedback-based learning about the emotional outcomes associated with each deck is critical to guide decisions towards safe options (Bechara et al, 2005). It is also consistent with previous evidence of drug-use interactions with the $s / s$ genotype impacting emotion-based impulsive decision-making (Roiser et al, 2005). The link between cannabis use and serotonin transporter function is supported by evidence that high doses of cannabinoid agonists induce reduction of platelet serotonin concentrations, accompanied by alterations of the functioning of the serotonergic system in cannabis chronic smokers (Velenovská and Fisar, 2007). There is also evidence that the proposed link between cannabis use and depression is only present in cannabis users carrying the $s / s$ genotype (Otten and Engels, 2011). These findings are in agreement with the notions that: (i) cannabis use impacts serotonin function; and (ii) this interaction may exacerbate behavioral vulnerabilities associated with the $s / s$ genotype. In this respect, recent studies have identified different-but related-behavioral phenotypes associated with the $s / s$ genotype, including negative emotionality and hypervigilance (Homberg and Lesch, 2011). Overall, the evidence suggest that cannabis use may exacerbate $s / s$ genotype-related behavioral phenotypes, such as negative emotionality or hypervigilance, which hamper the ability of cannabis users to learn adequately from negative feedback in the IGT.

In summary, we showed that cannabis users carrying the COMT val/val genotype exhibit lower accuracy of sustained attention than controls carrying the same genotype, that cannabis users val carriers commit more shifting errors than cannabis users met/met carriers, and that cannabis users carrying the SERT $s / s$ genotype have poorer decisionmaking than controls carrying the same genotype. This study holds a number of important strengths and also limitations worth noting. Strengths include the thorough control of potential confounders associated with the effects of cannabis use and genetic makeup. These confounders were addressed first during the process of recruitment and assessment of inclusion/exclusion criteria, in which we excluded individuals with comorbid substance-use or psychiatric disorders (eg, psychosis), and then in statistical analyses, in which we controlled for potential effects of age, gender, IQ, and smoking. In addition, we ruled out potential effects of acute cannabis use and other illegal drugs use by means of combined urine and hair toxicological analyses and medical control of withdrawal effects. An additional strength was the representativeness of the sample, which extracted a large number of non-treatment-seeking daily cannabis users and matched non-drug user controls. As for the limitations, we should first acknowledge that our findings apply to small subsets of cannabis using individuals ( $v a l$ and $s / s$ carriers), whereas we did not find main effects of any of the genotypes, and could not perform combined haplotype analyses. Nonetheless, our results inform about the molecular mechanisms by which cannabis use may exert a negative impact on executive functions, and they may entail important clinical implications. For example, the COMT inhibitor tolcapone has been proposed as a promising treatment for marijuana dependence (Weinstein and Gorelick, 2011), and its effect should be equivalent to a phenocopying of subjects' carriers of the $\mathrm{val} / \mathrm{val}$ genotype to the met/met genotype, resulting in more sustained dopamine brain concentrations. Therefore, the poor performance of cannabis using val carriers may improve after this therapeutic approach. Because of the impact of the COMT val allele on the neurodegeneration of dopamine networks (Gennatas et al, 2012), future studies should also explore whether this gene variant is associated with accelerated cognitive aging in cannabis users. A second limitation we are aware of is the problem of the large number of multiple comparisons. However, we only corrected for multiple tests in case of pairwise group comparisons for a given cognitive task. The alternative option of globally applying the Bonferroni correction was 
discarded because the resulting critical significance level for each test would have been extremely small, thus overly controlling for Type-1 error, but largely increasing the probability for Type-2 error. An additional shortcoming was the lack of significant correlations between cannabis use and cognition. This may be due, along with other factors, to the relative homogeneity of cannabis use patterns among participants, thus reducing variability of distributions and opportunities to detect associations.

\section{ACKNOWLEDGEMENTS}

We are indebted to Nicolas Vienney and Joyce Carvalho de Lima Ferreira for their technical expertise. This study was supported by grants from the National Institutes of Health (NIH) Grant No. 1 R01 DA017987, Grant No. 2005SGR00032, Fondo de Investigaciones Sanitarias (FIS-00/00777), and DIUE de la Generalitat de Catalunya 2009 (Grant SGR 718). The funders had no role in study design, data collection and analysis, decision to publish, or preparation of the manuscript.

\section{DISCLOSURE}

The authors declare no conflict of interest.

\section{REFERENCES}

Arnone D, Barrick TR, Chengappa S, Mackay CE, Clark CA, AbouSaleh MT (2008). Corpus callosum damage in heavy marijuana use: preliminary evidence from diffusion tensor tractography and tract-based spatial statistics. NeuroImage 41: 1067-1074.

Barnes JJ, Dean AJ, Nandam LS, O'Connell RG, Bellgrove MA (2011). The molecular genetics of executive function: role of monoamine system genes. Biol Psychiatry 69: e127-e143.

Barnes SA, Young JW, Neill JC (2012). $\mathrm{D}_{1}$ receptor activation improves vigilance in rats as measured by the 5-choice continuous performance test. Psychopharmacology (Berl) 220: 129-141.

Batalla A, Soriano-Mas C, López-Solà M, Torrens M, Crippa JA, Bhattacharyya $S$ et al (2012). Modulation of brain structure by catechol-O-methyltransferase $\mathrm{Val}(158)$ Met polymorphism in chronic cannabis users. Addict Biol; advance online publication on 14 January 2013. doi:10.1111/adb.12027.

Bechara A, Damasio H, Tranel D, Damasio AR (2005). The Iowa Gambling Task and the somatic marker hypothesis: some questions and answers. Trends Cogn Sci 9: 159-162.

Bechara A, Tranel D, Damasio H (2000). Characterization of the decision-making deficit of patients with ventromedial prefrontal cortex lesions. Brain 123: 2189-2202.

Behan AT, Hryniewiecka M, O'Tuathaigh CM, Kinsella A, Cannon M, Karayiorgou M et al (2012). Chronic adolescent exposure to delta-9-tetrahydrocannabinol in COMT mutant mice: impact on indices of dopaminergic, endocannabinoid and GABAergic pathways. Neuropsychopharmacology 37: 1773-1783.

Besson M, Belin D, McNamara R, Theobald DE, Castel A, Beckett VL et al (2010). Dissociable control of impulsivity in rats by dopamine $\mathrm{d} 2 / 3$ receptors in the core and shell subregions of the nucleus accumbens. Neuropsychopharmacology 35: 560-569.

Bevilacqua L, Goldman D (2011). Genetics of emotion. Trends Cogn Sci 15: 401-408.

Bilder RM, Volavka J, Lachman HM, Grace AA (2004). The catechol-O-methyltransferase polymorphism: relations to the tonic-phasic dopamine hypothesis and neuropsychiatric phenotypes. Neuropsychopharmacology 29: 1943-1961.
Bolla KI, Brown K, Eldreth D, Tate K, Cadet JL (2002). Dose-related neurocognitive effects of marijuana use. Neurology 59: 1337-1343. Camí J, Farré M (2003). Drug addiction. N Engl J Med 349: 975-986. Chamberlain SR, Robbins TW, Winder-Rhodes S, Muller U, Sahakian BJ, Blackwell AD et al (2011). Translational approaches to frontostriatal dysfunction in attention-deficit/hyperactivity disorder using a computerized neuropsychological battery. Biol Psychiatry 69: 1192-1203.

Cuyas E, Verdejo-Garcia A, Fagundo AB, Khymenets O, Rodriguez J, Cuenca A et al (2011). The influence of genetic and environmental factors among MDMA users in cognitive performance. PLoS One 6: e27206.

de la Torre R, Civit E, Svaizer F, Lotti A, Gottardi M, Miozzo M (2010). High throughput analysis of drugs of abuse in hair by combining purposely designed sample extraction compatible with immunometric methods used for drug testing in urine. Forensic Sci Int 196: 18-21.

European Monitoring Center for Drugs and Drug abuse (2010): Annual Report 2010. Retrieved from http://www.emcdda.europa.eu/events/2010/annual-report.

Falcon M, Pichini S, Joya J, Pujadas M, Sanchez A, Vall O et al (2011). Maternal hair testing for the assessment of fetal exposure to drug of abuse during early pregnancy: comparison with testing in placental and fetal remains. Forensic Sci Int 218: 92-96.

Fontes MA, Bolla KI, Cunha PJ, Almeida PP, Jungerman F, Laranjeira RR et al (2011). Cannabis use before age 15 and subsequent executive functioning. Br J Psychiatry 198: 442-447.

Gennatas ED, Cholfin JA, Zhou J, Crawford RK, Sasaki DA, Karydas A et al (2012). COMT Val158Met genotype influences neurodegeneration within dopamine-innervated brain structures. Neurology 68: 1663-1669.

Grant I, Gonzalez R, Carey CL, Natarajan L, Wolfson T (2003). Non-acute (residual) neurocognitive effects of cannabis use: a meta-analytic study. J Int Neuropsychol Soc 9: 679-689.

Greenberg BD, Tolliver TJ, Huang SJ, Li Q, Bengel D, Murphy DL (1999). Genetic variation in the serotonin transporter promoter region affects serotonin uptake in human blood platelets. Am J Med Genet 88: 83-87.

Hamidovic A, Dlugos A, Palmer AA, de Wit H (2010). Catechol-Omethyltransferase val158met genotype modulates sustained attention in both the drug-free state and in response to amphetamine. Psychiatric Genet 20: 85-92.

He Q, Xue G, Chen C, Lu Z, Dong Q, Lei X et al (2010). Serotonin transporter gene-linked polymorphic region (5-HTTLPR) influences decision making under ambiguity and risk in a large Chinese sample. Neuropharmacology 59: 518-526.

Henquet C, Rosa A, Krabbendam L, Papiol S, Fananás L, Drukker $M$ et al (2009). An experimental study of catechol-omethyltransferase Val158Met moderation of delta-9-tetrahydrocannabinol-induced effects on psychosis and cognition. Neuropsychopharmacology 31: 2748-2757.

Hermann D, Leménager T, Gelbke J, Welzel H, Skopp G, Mann K (2009). Decision making of heavy cannabis users on the Iowa Gambling Task: stronger association with THC of hair analysis than with personality traits of the Tridimensional Personality Questionnaire. Eur Addict Res 15: 94-98.

Homberg JR, Lesch KP (2011). Looking on the bright side of serotonin transporter gene variation. Biol Psychiatry 69: 513-519.

Homberg JR, van den Bos R, den Heijer E, Suer R, Cuppen E (2008). Serotonin transporter dosage modulates long-term decision-making in rat and human. Neuropharmacology 55: $80-84$.

Jager G, Block RI, Luijten M, Ramsey NF (2010). Cannabis use and memory brain function in adolescent boys: a cross-sectional multicenter functional magnetic resonance imaging study. $\mathrm{J} \mathrm{Am}$ Acad Child Adolesc Psychiatry 49: 561-572.

Jager G, Kahn RS, Van Den Brink W, Van Ree JM, Ramsey NF (2006). Long-term effects of frequent cannabis use on working 
memory and attention: an fMRI study. Psychopharmacology (Berl) 185: 358-368.

Lyons MJ, Bar JL, Panizzon MS, Toomey R, Eisen S, Xian H et al (2004). Neuropsychological consequences of regular marijuana use: a twin study. Psychol Med 34: 1239-1250.

Nolan KA, Bilder RM, Lachman HM, Volavka J (2004). Catechol O-methyltransferase Val158Met polymorphism in schizophrenia: differential effects of $\mathrm{Val}$ and Met alleles on cognitive stability and flexibility. Am J Psychiatry 161: 359-361.

O'Tuathaigh CM, Hryniewiecka M, Behan A, Tighe O, Coughlan C, Desbonnet L et al (2010). Chronic adolescent exposure to $\Delta-9$ tetrahydrocannabinol in COMT mutant mice: impact on psychosis-related and other phenotypes. Neuropsychopharmacology 35: 2262-2273.

Otten R, Engels RC (2011). Testing bidirectional effects between cannabis use and depressive symptoms: moderation by the serotonin transporter gene. Addict Biol; advance online publication on 4 October 2011. doi:10.1111/j.1369-1600.2011. 00380.x.

Pattij T, Wiskerke J, Schoffelmeer AN (2008). Cannabinoid modulation of executive functions. Eur $J$ Pharmacol 585: 458-463.

Pichini S, Poudevida S, Pujadas M, Menoyo E, Pacifici R, Farre M et al (2006). Assessment of chronic exposure to MDMA in a group of consumers by segmental hair analysis. Therap Drug Monit 28: 106-109.

Robbins TW (2007). Shifting and stopping: fronto-striatal substrates, neurochemical modulation and clinical implications. Philos Trans R Soc Lond Ser B 362: 917-932.

Robbins TW, James M, Owen AM, Sahakian BJ, Lawrence AD, McInnes L et al (1998). A study of performance on tests from the CANTAB battery sensitive to frontal lobe dysfunction in a large sample of normal volunteers: implications for theories of executive functioning and cognitive aging. Cambridge Neuropsychological Test Automated Battery. J Int Neuropsychol Soc 4: 474-490.

Robbins TW, James M, Owen AM, Sahakian BJ, McInnes L, Rabbitt P (1994). Cambridge Neuropsychological Test Automated Battery (CANTAB): a factor analytic study of a large sample of normal elderly volunteers. Dementia 5: 266-281.

Robertson IH, Garavan H (2000). Vigilant attention. In: Gazzaniga M (ed) The New Cognitive Neurosciences. MA: MIT Press: Cambridge, MA, USA563-578.

Roiser JP, Cook LJ, Cooper JD, Rubinsztein DC, Sahakian BJ (2005). Association of a functional polymorphism in the serotonin transporter gene with abnormal emotional processing in ecstasy users. Am J Psychiatry 162: 609-612.

Sonuga-Barke EJ, Kumsta R, Schlotz W, Lasky-Su J, Marco R, Miranda A et al (2011). A functional variant of the serotonin transporter gene (SLC6A4) moderates impulsive choice in attention-deficit/hyperactivity disorder boys and siblings. Biol Psychiatry 70: 230-236.

Substance Abuse and Mental Health Services Administration (SAMHSA) (2010): National Survey on Drug Use and Health 2010. Retrieved from https://nsduhweb.rti.org/.

Tan HY, Chen Q, Goldberg TE, Mattay VS, Meyer-Lindenberg A, Weinberger DR et al (2007). Catechol-O-methyltransferase Val158Met modulation of prefrontal-parietal-striatal brain systems during arithmetic and temporal transformations in working memory. J Neurosci 27: 13393-13401.

Torrens M, Serrano D, Astals M, Perez-Dominguez G, MartinSantos R (2004). Diagnosing comorbid psychiatric disorders in substance abusers: validity of the Spanish versions of the Psychiatric Research Interview for Substance and Mental Disorders and the Structured Clinical Interview for DSM-IV. Am J Psychiatry 161: 1231-1237.

Tunbridge EM, Bannerman DM, Sharp T, Harrison PJ (2004). Catechol-o-methyltransferase inhibition improves set-shifting performance and elevates stimulated dopamine release in the rat prefrontal cortex. J Neurosci 24: 5331-5335.

Vaidya JG, Block RI, O’Leary DS, Ponto LB, Ghoneim MM, Bechara A (2011). Effects of chronic marijuana use on brain activity during monetary decision-making. Neuropsychopharmacology 37: 618-629.

van den Bos R, Homberg J, Gijsbers E, den Heijer E, Cuppen E (2009). The effect of COMT Val158 Met genotype on decisionmaking and preliminary findings on its interaction with the 5-HTTLPR in healthy females. Neuropharmacology 56: 493-498.

Velenovská M, Fisar Z (2007). Effect of cannabinoids on platelet serotonin uptake. Addict Biol 12: 158-166.

Verdejo-Garcia A, Benbrook A, Funderburk F, David P, Cadet JL, Bolla KI (2007). The differential relationship between cocaine use and marijuana use on decision-making performance over repeat testing with the Iowa Gambling Task. Drug Alcohol Depend 90: 2-11.

Verdejo-García A, Pérez-García M (2007). Profile of executive deficits in cocaine and heroin polysubstance users: common and differential effects on separate executive components. Psychopharmacology (Berl) 190: 517-530.

Watter S, Geffen GM, Geffen LB (2001). The n-back as a dual-task: P300 morphology under divided attention. Psychophysiology 38: 998-1003.

Weinstein AM, Gorelick DA (2011). Pharmacological treatment of cannabis dependence. Curr Pharm Des 17: 1351-1358.

Yücel M, Solowij N, Respondek C, Whittle S, Fornito A, Pantelis C et al (2008). Regional brain abnormalities associated with longterm heavy cannabis use. Arch Gen Psychiatry 65: 694-701.

Zalesky A, Solowij N, Yücel M, Lubman DI, Takagi M, Harding IH et al (2012). Effect of long-term cannabis use on axonal fibre connectivity. Brain 135: 2245-2255.

Supplementary Information accompanies the paper on the Neuropsychopharmacology website (http://www.nature.com/npp) 